\title{
Histomorphological Study of Gestational Trophoblastic Lesions in a Tertiary Medical Centre: A Prospective Study
}

\author{
Nidhi Rajendra ${ }^{1 *}$, Fouzia Kauser ${ }^{2}$, Prashanth Madapura V ${ }^{3}$, Doddikoppad.M M ${ }^{4}$ \\ ${ }^{1}$ Department of Pathology, Dr. B R Ambedkar Medical College and Research Centre, KG Halli, Bangalore, Karnataka, India \\ ${ }^{2}$ Department of Pathology, Clumax Diagnostics, The Banyan Apartments, Anjanapura, JP Nagar 9th Phase, Bangalore. India \\ ${ }^{3}$ Department of Paediatrics, M S Ramaiah Medical College and Hospital, MSRIT Post, Bangalore, India \\ ${ }^{4}$ Department of Pathology, Basaveshwara Medical College and Hospital, Chitradurga, India.
}

\begin{abstract}
Background: Gestational trophoblastic diseases(GTDs) consists of pregnancy related disorders ranging from benign Hydatidiform mole, Invasive mole to neoplastic conditions including Choriocarcinoma, Placental site trophoblastic tumor and Epitheloid trophoblastic tumor with varying potential for local invasion and metastasis. GTDs mimic growth pattern encountered in early normal placental development, nonmolar abortions and variety of nontrophoblastic lesions. Therefore an appreciation of different types of GTDs with its histomorphological manifestations are important for the confirmation of diagnosis. Thus the study was undertaken.
\end{abstract}

Materials \& Methods: The material for the study comprised of products of conception specimens received in the Department of Pathology, J.J.M. Medical college, Davangere, India during the period of 2 years. Detailed gross examination was done before fixation and subsequent microscopic analysis was done.

Results: 65 cases of GTDs were diagnosed in 495 cases of products of conception. 37 cases of complete mole, 27 cases of partial mole and one case of Invasive mole were diagnosed. Diffuse hydropic swelling of the villi, round smooth villous outline, circumferential trophoblastic proliferation and focal hydropic swelling of the villi, irregular scalloping outline of the enlarged villi, focal trophoblastic cell proliferation were characteristic features of complete \& partial mole respectively. Histomorphological features were analysed in all the cases and compared with studies done by others.

Conclusion: The classification and histomorphological analysis of GTDs are important elements for better understanding of the disease. Studies are needed to look for ancillary markers in distinguishing different types of GTDs and for predicting the prognosis.

Keywords: Gestational Trophoblastic Diseases, Complete Mole, Partial Mole, Invasive Mole.

\section{Introduction}

Gestational trophoblastic lesions (GTD) includes a heterogenous family encompassing various neoplastic and non neoplastic lesions arising from different type of villous and non villous trophoblast. ${ }^{[1]}$ Trophoblast is an integral component of the human placenta for mediating the implantation of the embryo, protecting the fetus from the maternal immune system, delivering nutrient and removing waste products as well as producing vital pregnancy hormones. ${ }^{[1]}$ Hertig referred to gestational trophoblastic neoplasms as "God's first cancer and man's first cure". ${ }^{[2]}$

Gestational trophoblastic disease constitutes a diverse group of lesions that includes Hydatidiform mole, benign non neoplastic lesions, and gestational trophoblastic neoplasms ${ }^{[3]}$

Gestational trophoblastic lesions arises from trophoblast, a tissue normally exhibiting features otherwise associated with malignant tumors like intense proliferation, anaplasia, infiltration of contiguous structures, invasion of blood vessels and hematogenous dissemination. Thus it may be difficult to discriminate benign, even normal proliferating trophoblastic tissue from its malignant counterpart on morphologic grounds alone. ${ }^{[4]}$

Multiple hCG related molecules are present in serum and urine samples of pregnancy and trophoblastic disease. These include degraded molecules, hyper glycosylated molecules, large subunits and their fragments, which are estimated in serum and urine using different combination of antibodies. While all are appropriate for normal pregnancy application, only certain types of assay may be correct for monitoring trophoblastic diseases. ${ }^{[5,6]}$ With a half life of 24-36 hours, hCG is the most sensitive and specific marker for trophoblastic tissue. Current methods for hCG measurement do not reliably discriminate among pregnancy, GTD and non gestational trophoblastic tumors. However serial measurements of hCG have revolutionized the management of GTD for several reasons. The amount of hCG produced correlates with tumor volume so that a serum hCG of $5 \mathrm{IU} / \mathrm{lt}$ corresponds to approximately 104 
- 105 viable tumor cells. Consequently, these assays are several orders of magnitude more sensitive than the best imaging modalities available today. The hCG levels can be used to determine prognosis. Serial measurements allow progress of the disease or response to therapy to be monitored. ${ }^{[7,8,9]}$

Gestational trophoblastic diseases mimic growth pattern encountered in early normal placental development, non molar abortions and a variety of nontrophoblastic lesions, therefore an appreciation of histomorphological manifestations of gestational trophoblastic lesions are important to avoid confusing with their mimickers. Thus the study is undertaken for histomorphological evaluation.

\section{Material and Methods}

This is a prospective study done during the period from July 2009 - June 2011 (2 years) in the department of Pathology, J.J.M Medical college Davangere, India. Brief clinical history and examination findings were obtained from treating Obstetrician.

During the study period, total of 12188 specimens were received in the histopathology section, in the department of Pathology. Among these, 495 were products of conception. Gestational trophoblastic lesions were identified in 65 cases and included in this study. Serum $\beta$ hCG level estimation was done during pre evacuation wherever it was possible. Permission and clearance was obtained from Institution's Ethical and scientific committee. Written consent was obtained from each individual who were included in the study group.

Inclusion Criteria: All specimens received at the Department of Pathology, J.J.M Medical college, Davangere showing trophoblastic tissue excluding term placenta during the span of 2 years(from July 2009- June 2011)

Exclusion Criteria: Non gestational trophoblastic lesions.

\section{Results}

Sixty five GTD cases were histopathologically diagnosed in the present study and were classified according to WHO classification criteria. Serum $\beta$ hCG level estimation ( single laboratory) was possible in 55 cases(Table 1: Mean serum $\beta$ hCG levels done pre-evacuation) and ultrasound abdomen was done in all 65 cases. There were 37 cases of complete mole, 27 cases of partial mole and one case of Invasive mole. Of the total 65 cases of GTDs, 8 cases had previous history of abortions.

\section{Table 1: Mean serum $\beta$ hCG levels (done pre-evacuation)}

Serum $\beta$ hCG levels were done by in all suspected GTD cases before evacuation of products of conception in a single laboratory. Out of 65 GTD cases, serum $\beta$ hCG levels reports were available in 55 cases. Serum $\beta$ hCG levels reports in 7 cases of complete hydatidiform mole and 3 cases of partial hydatidiform mole was not done. We sorted out the serum $\beta$ hCG levels based on their gestational age ( in weeks) at which it was done and also the type of GTD.

The mean values of the serum $\beta$ hCG levels in that particular gestational age was found (table-1). The serum $\beta$ hCG levels when plotted on the bar chart (y axis) against gestational age ( $\mathrm{x}$ axis) at which the values were done (graph-1). In graph it is evident that mean serum $\beta$ hCG levels in complete mole was always on the higher side than in partial mole at that respective gestational age.

Gross Features of GTD: Gross specimens were subjected for detailed examination to identify fetal parts. No fetal parts were observed in all suspected cases. All 65 cases showed multiple grape like vesicles and edematous change. The size of the specimens varied from $2 \mathrm{~cm}$ to 15 cms. Vesicles were clear fluid filled, smooth surface, cystic in consistency and the color varied from grey white to grey brown. In order to appreciate the vesicles better, they were separated from blood clots and kept in normal water and photography was taken. Then the tissue was fixed in formalin. Specimens were subjected for detailed gross examination to identify the fetal parts.

Complete Mole: Out of 37 cases of complete mole, 34 cases demonstrated diffuse hydropic swelling of the villi and circumferential trophoblastic proliferation. All cases showed round smooth villous outline. Three cases of early complete mole demonstrated focal trophoblastic proliferation without hydropic changes in the villi. In complete mole villi were of various sizes and even the smaller villi also showed edematous changes. The proliferating cytotrophoblast and syncytiotrophoblast showed circumferential growth from the villous surface. Mild to moderate degree of trophobastic proliferation was noted. 17 cases showed central cistern formation characterized by a prominent central space that is entirely acellular fluid filled spaces within the centre of the villi. 27 cases had myxoid intravillous stroma, 4 cases had hydropic intravillous stroma and 6 cases showed both myxoid and hydropic stroma.10 cases showed trophoblastic inclusions. Areas of haemorrhage and necrosis was seen in 15 cases. Decidual cells were seen in 22 cases. None of these cases showed blood vessels within the intravillous stroma or any embryonic tissue. 3 cases showed features of early complete mole characterized by redundant bulbous terminal villi, hypercellular villous stroma with primitive stellate cells and karyorrhexia, a labyrinthine network of villous stromal canaliculi and focal hyperplasia of cytotrophoblast and syncytiotrophoblast. 
Partial Mole: 27 cases were diagnosed as partial hydatidiform mole $\&$ all cases exhibited focal hydropic swelling and small normal sized villi along with irregular and scalloping outline of the enlarged villi. Focal proliferation of cytotrophoblast and syncytiotrophoblast cells was seen in all 27 cases of partial hydatidiform mole. Trophoblastic hyperplasia is less marked, focal, consisting of small, haphazard tufts of trophoblast, often syncytiotrophoblast, emanating from the surface of some of the abnormal villi. All 27 cases of partial mole had scalloped outline of the enlarged villi, yielding a pattern of trophoblastic invaginations into the villous stroma. 23 cases showed trophoblastic inclusions. Central cisterns are less conspicuous and present in 4 cases of partial mole. Fibrotic intravillous stroma was seen in 17 cases, myxoid intravillous stroma was seen in 8 cases and hydropic intravillous stroma was seen in 2 cases. Blood vessels was seen in 12 cases and were devoid of nucleated red blood cells. Intermediate trophoblasts in the intervillous spaces was seen in 8 cases. Decidual cells were seen in 14 cases and focal areas of haemorrhage was present in 10 cases.

Partial mole should be distinguished from hydropic abortus. The hydropic abortus usually are smaller specimens. Villi in hydropic abortus are enlarged only slightly and do not assume the large dimensions found in complete or partial mole. In hydropic abortus the villous swelling and central cistern formation are not present. The villous trophoblast is attenuated and trophoblastic atypia is absent or minimal. The trophoblast proliferating from the villous surface of an abortus shows polar distribution characterized by proliferation of trophoblast at the distal end of the villous that implants into the basal plate.

Invasive Mole: This was seen in a multiparous woman. Her pre-operative serum $\beta$ hCG levels was $126000 \mathrm{mIU} /$ $\mathrm{ml}$. Total hysterectomy specimen measuring $9 \times 7 \times 5 \mathrm{cms}$. Small grape like vesicles altogether measuring $5 \times 4 \mathrm{cms}$ were present within the endometrial cavity invading upto the serosa but not rupturing the uterus. The vesicles were small measuring $<1 \mathrm{~cm}$ in size and were adherent to endometrial cavity. This case of invasive mole was diagnosed in a woman aged 39 yrs, which was thought to be preceded by Hydatidiform Mole. Microscopically it showed villi of varying sizes, lined by proliferating cytotrophoblasts and syncytiotrophoblasts. Transmural invasion of the myometrium by the villi was seen. Villi were enlarged, irregular in size and shape, with hydropic degeneration in the stroma. No mitosis was seen. Focal areas of haemorrhage and necrosis was seen. There was presence of molar villi and trophoblast cells deep in the myometrium.

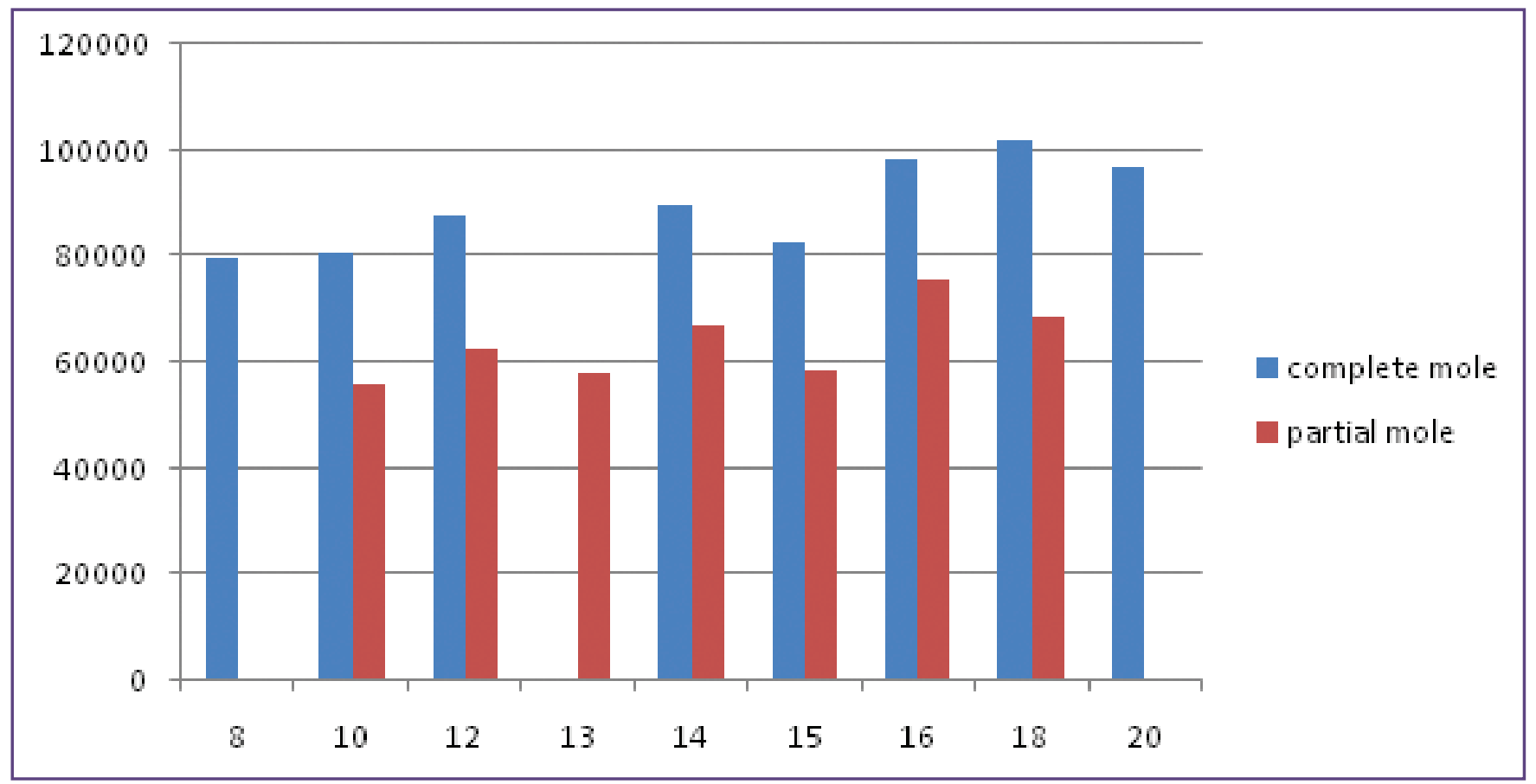

Graph 1: Mean serum $\beta$ hCG levels complete mole and partial mole. 
Table 1: Mean serum $\beta$ hCG levels (done pre-evacuation).

\begin{tabular}{|l|l|l|}
\hline Gestational age (weeks) & $\begin{array}{l}\text { Mean serum } \beta \text { hCG levels in complete } \\
\text { mole }(\mathrm{mlU} / \mathrm{ml})\end{array}$ & $\begin{array}{l}\text { Mean serum } \beta \text { hCG levels in } \\
\text { partial mole (mlU/ml) }\end{array}$ \\
\hline 8 & 79460 & 40100 \\
\hline 10 & 85650 & 56060 \\
\hline 12 & 87642 & 62598 \\
\hline 13 & 89610 & 57826 \\
\hline 14 & 82697 & 67006 \\
\hline 16 & 98213 & 58322 \\
\hline 18 & 101800 & 75450 \\
\hline 20 & 97120 & 68460 \\
\hline
\end{tabular}

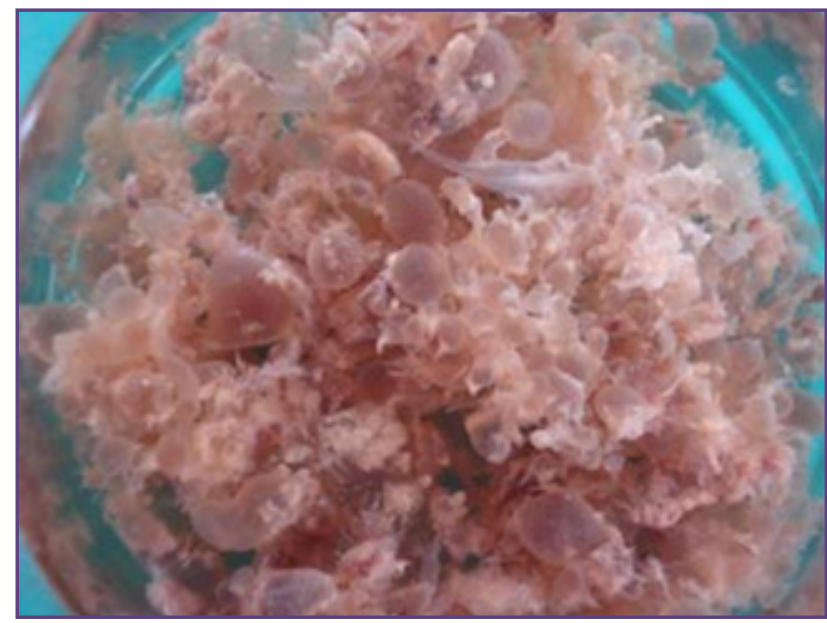

Fig.1: Gross photograph showing edematous villi of varying sizes with membranous tissue.

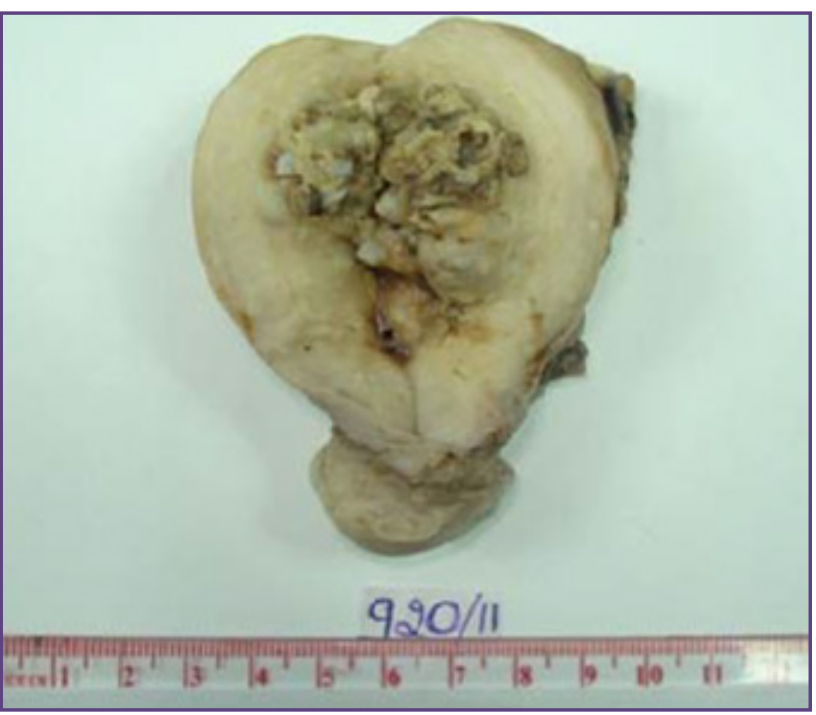

Fig. 3: Invasive mole - Uterine cavity completely fiilled with multiple grape like vesicles.

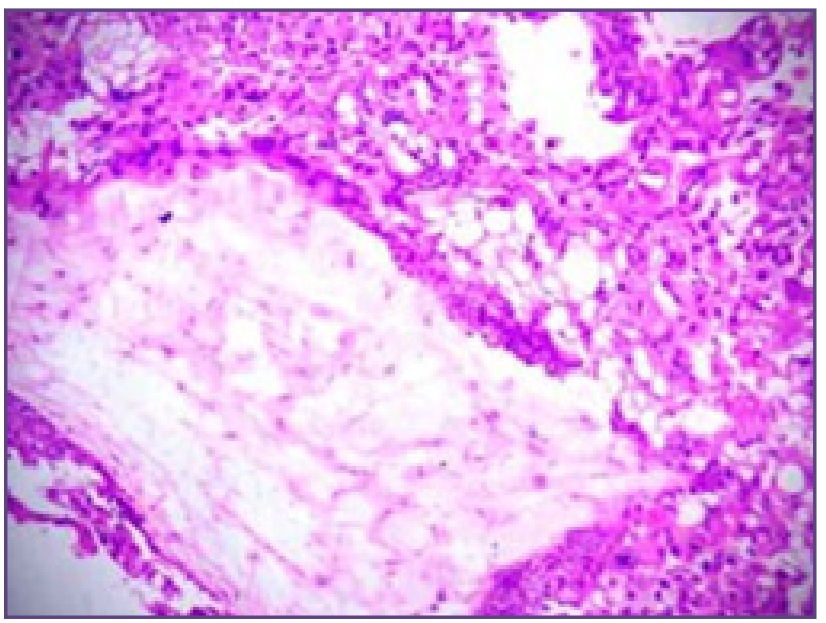

Fig. 2: Edematous villi showing circumferential trophoblastic proliferation with myxoid intravillous stroma.(H\&E 4x).

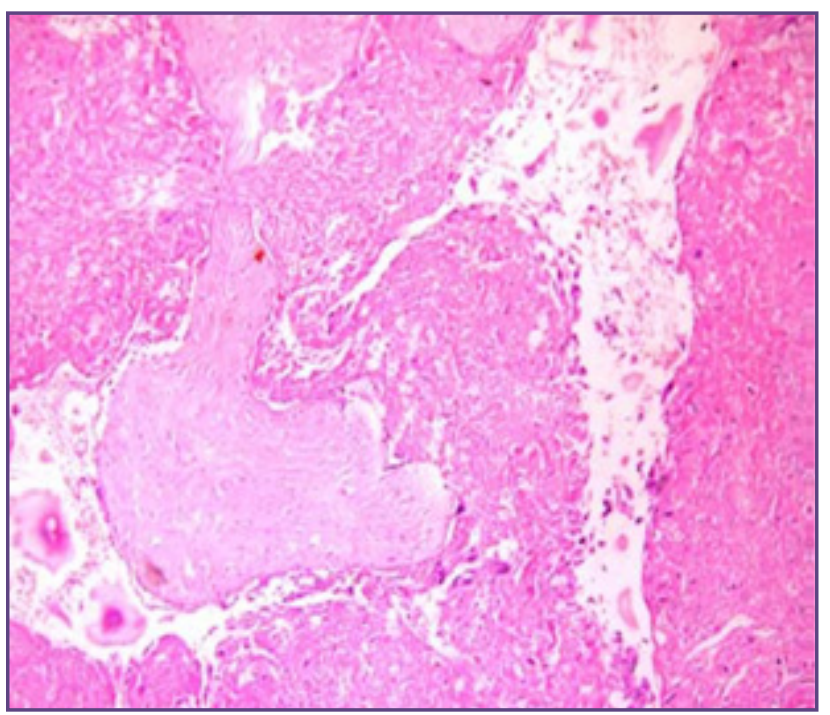

Fig.4: Chorionic Villi present within the myometrium. 


\section{Discussion}

Gestational trophoblastic lesions represents a spectrum of lesions characterized by an abnormal proliferation of trophoblasts which includes complete mole, partial mole, invasive mole, choriocarcinoma and placental site trophoblastic tumors. Some of these lesions are true neoplasms where as others represents abnormally formed placentas with a predisposition for neoplastic transformation of trophoblasts.

In the study period of two years, among 12188 pathological specimens, $4.06 \% \quad(495 \mathrm{cases})$ were products of conception. We analysed 495 specimens of products of conception, received in our department for histopathological analysis. Of 495 specimens, 65 cases were diagnosed as GTD's based on morphology and quantitative serum $\beta$ hCG level estimation .

All 34 cases of complete mole were characterized by diffuse hydropic swelling of the villi with round smooth villous outline and circumferential trophoblastic proliferation. A study conducted in a tertiary care hospital, Lahore on histopathological analysis of compete mole and partial mole in 2011 June stated that all cases of complete mole demonstrated diffuse swelling of the villi and exhibited circumferential proliferation of the trophoblast. ${ }^{[10]}$ Another study done in Tokyo also defined $100 \%$ existence of villous edema in complete mole and also in partial mole. ${ }^{[1]}$ Similar reports were seen in other studies ${ }^{[12]}$ Study done by Richard $\mathrm{M} \mathrm{C}$ et al revealed that $65 \%$ of complete mole revealed uniform dilation of the villi and $35 \%$ had focal dilation. ${ }^{[13]}$

The histomorphological features of 27 cases of partial mole were focal hydropic swelling of the villi, scalloping villous outline and focal trophoblastic proliferation and trophpblastic hyperplasia was less marked than that of complete mole. In a study done by Fukunaga et al on partial mole described that focal villous edema, focal syncytiotrophoblastic hyperplasia, villous scalloping outline, vacuolated syncytiotrophoblasts and trophoblastic inclusions were seen in all $(100 \%)$ the cases of partial mole. ${ }^{[11]}$

In a study done by Richard MC et al demonstrated focal dilation of the villi in $86 \%$, scalloped villi in $83 \%$ and uniform dilation of the villi in only $10 \%$ of partial mole. ${ }^{[13]}$ Trophoblastic inclusions are nothing but break in the continuity of outline of the villi and was seen in $27 \%$ ( 10 cases) of complete mole. In a study done by Rozina et al and Richard MC et al, the incidence of trophoblastic inclusions in complete mole was $92.5 \%$ and $65 \%$ respectively. ${ }^{[10,13]}$ Thus presence of trophoblastic inclusion is not a uniform feature. But in case of partial mole, trophoblastic inclusions were found in $85.5 \%$ (23 cases) which can be attributed to commonly encountered scalloped margins which forms pseudo inclusions within the stroma. In a study done by Fukunaga et al, trophoblastic inclusions was seen in 100\% of partial mole. ${ }^{[11]}$ Richard MC et al described trophoblastic inclusions in $79 \%$ of the partial mole. ${ }^{[13]}$ Thus presence of trophoblastic inclusions is a more consistent finding in partial mole than complete mole.

Marked central cistern formation are predominant feature of complete mole which is attributed to widely separated broken strands of fibrillar material. Central cistern was seen in $50 \%$ in complete mole and $14.8 \%$ in partialmole in our study. Richard MC and others described $100 \%$ presence of central cisterns in both complete mole and partial mole. ${ }^{[13]}$ However Fukunaga and others described in a different way, according to them $93 \%$ of partial mole had central cistern formation and central cistern was occasionally observed in complete mole. ${ }^{[11]}$ Thus there exists different views about the central cistern formation .

We found myxoid intravillous stroma in $72.9 \%$ (27 cases) of complete mole and $29.6 \%(8$ cases $)$ of partial mole and fibrotic intravillous stroma was described in $62.9 \%$ which was seen only in partial mole. This finding was consistent with the study done by Fukunga and others. In their study,intravillous fibrous stroma was seen in $57 \%$ of partial mole. ${ }^{[11]}$

In our study, hydropic intravillous stroma was seen in less than $10 \%$ (4 cases) of complete mole and partial mole. Myxoid and hydropic intravillous stroma was seen in $16.2 \%$ (6 cases) of complete mole only. Intermediate trophoblasts in the intervillous space were seen in $40.5 \%$ ( 15 cases) of complete mole and $29.6 \%$ ( 8 cases) of partial mole. Blood vessels in the stroma were described in $44.4 \%$ ( 12 cases) of partial mole which were devoid of nucleated red blood cells. Fukunga and others described presence of blood vessels in $89 \%$ cases in partial mole. ${ }^{[11]}$ Rozina Jaffer and others described a similar finding about the presence of blood vessels in $65 \%$ cases of partial mole. ${ }^{[10]}$ Similar findings was observed in a study done by Young Ho De and others ${ }^{12}$. Blood vessels were absent in complete mole in our study. Similar finding was seen in other studies. ${ }^{[10,12]}$

One case of invasive mole in 39 year old woman with gravid-5 presented with 2 months amenorrhoea and bleeding per vagina. The incidence of invasive mole was $1.53 \%$. A study from Pakistan done in 2009, revealed a very high incidence of invasive mole $(23.3 \%),{ }^{[14]}$ where the frequency of GTD was 28 per 1000 live birth which was alarmingly high. In another pathological study done in North India in 2007, revealed that the incidence of 
invasive mole was $8.7 \% .{ }^{[15]}$ In our study, microscopically invasive mole showed villi of varying sizes lined by cytotrophoblasts and syncytiotrophoblast and were invading the myometrium. Transmural invasion of the myometrium by the viili was a similar finding to other studies. ${ }^{[16,17]}$ The diagnosis of invasive mole rests on the demonstration of complete hydatidiform mole invading the myometrium or the presence of villi in the metastatic lesion. ${ }^{[16,17]}$

3 cases of early complete mole were diagnosed in our study which were characterized by redundant bulbous terminal villi, hypercellular villous stroma with primitive stellate cells and karyorrhexia, a labyrinthine network of villous stromal canaliculi and focal hyperplasia of cytotrophoblast and syncytiotrophoblast. Few studies suggested that complete vasculogenic differentiation is significantly retarded in very early complete mole, due to increased apoptosis in the precursor components of blood vessels. It may result in a lack of vascular drainage and cause progressive accumulation of vesicular fluid in the later gestational period. ${ }^{[18]}$ Another study from the developed country revealed that bulbous villous pattern ,cellular stroma and focal trophoblastic proliferation are characteristic features of early complete mole. ${ }^{[19]}$

Many histological features in early complete mole are shared with early developmental stage of normal villous stroma, but timely differentiation of stromal components is defective or arrested and immature features are for prolonged periods. These findings confirm that hydatidiform mole is not only a disease of trophoblastic proliferation, but is associated with the abnormal or incomplete maturation of villous stromal components. These findings were also reflected in a study done by Kyo.R.K et al. ${ }^{[20]}$

Estimation of $\beta$ hCG levels may be of value in diagnosing molar pregnancies. The clinical significance of the accurate detection of GTD is in relation to development of persistent gestational trophoblastic neoplasm(pGTN), which is defined as either clinically apparent invasive disease or persistently raised serum human chorionic gonadotropin (hCG) concentration due to gestational trophoblastic proliferation. ${ }^{[2]}$ In women with a complete mole, the quantitative serum $\beta$-hCG level is higher than expected, often exceeding 100,000 IU/L. In case of a partial mole, the level of $\beta$-hCG is often within the wide range associated with normal pregnancy and the symptoms are usually less pronounced. For these reasons the diagnosis of a partial mole is often missed clinically and made from subsequent histologic assessment of the abortive material. ${ }^{[22]}$

In our study the pre evacuation mean serum $\beta$ hCG levels were higher in GTDs when compared to the $\beta$ hCG normogram levels. ${ }^{[23]}$ It is also evident that mean serum $\beta$ hCG levels in complete mole was always on the higher side than in partial mole at that respective gestational age.

\section{Conclusion}

Frequency of complete hydatidiform mole was higher as compared to partial hydatidiform mole. Histomorphological features of complete hydatidiform mole differs from partial hydatidiform mole on the basis of trophoblastic proliferation, villous contours, scalloping borders and central cisterns. The mean serum $\beta$ hCG levels in complete hydatidiform mole was more than the partial hydatidiform mole at that gestational age. Further studies are needed to prove this. Studies are needed to look for ancillary markers in distinguishing complete hydatidiform mole and partial hydatidiform mole.

Ethical Approval : The study was approved by institution ethical and scientific committee.

\section{References}

1. Annie NY Chung. Gestational trophoblastic disease. In : Stanley JR, George LM, eds. Robboy's pathology of female reproductive tract. Churchill Livginstone: Elsevier (Pub); 2009: 881-907.

2. Hertig AT. Human trophoblast coated by Charles B Hammond. Gestational trophoblastic neoplasia, history of current understanding. Obst \& Gynec Clin of N-Am 1988;153:435-439.

3. Ie M S Michael TM, Robert JK. Gestational trophoblastic disease. In : Stacey EM, Jode KG, Harold AO, Victor R, Mart HS eds. Sternberg's Diagnostic surgical pathology. Lippincot Williams and Wilkins (pub) 2010. pp2049-69

4. Liane D, Shirley GD, Donald PG. Gestational trophoblastic neoplasm. Morphology correlates of therapeutic response. Am J Obstet Gynec 1978;130(7):801-806.

5. Laurnence A. Cole. hCG, its free subunits and its metabolites role in pregnancy and trophoblastic disease. J Reprod Med 1998;43:3-10.

6. Michael JS, Neil SS, Ross SB. Gestational trophoblastic disease. Lancet 2010;376:717-729.

7. Michael JS, and Edward SN. Management of gestational trophoblastic disease. In: David MG, Gillian T, William PM, Martin G, Michael AQ eds. Gynecologic cancer . Churchill Livginstone: Elsevier (Pub); 2004:555-573.

8. George AH, Carl MH. Ectopic pregnancy. In: Larry JC eds. Text book of gynecology. W.B. Saunder's Compnay; 1993 :242-260.

9. Ross SB, Donald PG. Gestational trophoblastic disease. In: Jonathan SB eds. Berek and Novak's gynecology. Wolters Kluwer Health and Lippincott Williams and Wilkins 2006 pp.1581-1603

10. Rozina J, Rahat K, and Asmaa Q. Histopathological review of partial and complete hydatidiform moles in a tertiary care hospital, Lahore Pakistan, Biomedica 2011 June;27:76-80. 
11. Fukunaga M, Shinichiro U and Yasuhiko E. Incidence of hydatidiform mole in a Tokyo Hospital : A 5 year (1989 to 1993) prospective, morphological and flow cytometric study. Human Pathol 1995;26(7):758-764.

12. Young HY, Hyun MK, Tchan KP, Chang KK and Yoo BL.Comparative cytogenetic and clinicopathologic studies on gestational trophoblastic neoplasia, especially hydatidiform mole. Yonsei Med J 1986;27(4):250-260.

13. Richard MC, Charles LH, Edwina JP, Henry JN, William F. Mc. Diagnostic considerations in molar gestations. Hum Pathol 1993;24(1);41-48.

14. Khairunnisa N, Guljareen H, Nizamuddin M, Ambreen H. Gestational trophoblastic disease: Experience at Nawabshah Hospital. J Ayub MedColl Abbottabad 2009;21(1):94-97.

15. Shakuntala C, Ambreen Q. Gestational trophoblastic neoplasms with special reference to invasive mole. Obstet Gynecol India 2007;57(2):124-127.

16. Amandeep KA, Shashi G, Vikram M, Rajat G, Rajni G. Invasive mole presenting as acute abdomen. J K Science 2011;13(1):35-36.

17. Debarmita M, Napur M, Ram PD, Ranu RB, Amiya KB, Subhash B.Partial invasive molar pregnancy -2 case reports. Al Ameen J Med Sci 2010;3(1):91-93.
18. MiJK, KyuRK, JaeYR, JaniceML, HyangIL. Diagnostic and pathogenetic significance of increased stromal apoptosis and incomplete vasculogenesis in complete hydatidiform moles in very early pregnancy periods. Am J Surg Pathol 2006;30(3):362-69.

19. David K. Michael VZ, Terry H and Raymond WR. Very early complete hydatidiform mole. Hum Pathol 1996;27:708-713.

20. Kyu RK, Bong HP, Young OKH, Hyuck CK and Robboy SJ. The villous stromal constituents of complete hydatidiform mole differ histologically in very early pregnancy from the normally developing placenta. Am J Surg Pathol 2009;33:176-185.

21. Sebire.NJ.The diagnosis of gestational trophoblastic disease in early pregnancy: implications for screening, counseling and management Ultrasound Obstet Gynecol 2005; 25: 421-424.

22. Soper JT, Mutch DG, Schink JC. American College of Obstetricians and Gynecologists. Diagnosis and treatment of gestational trophoblastic disease: ACOG Practice Bulletin No. 53. Gynecol Oncol. 2004 Jun;93(3):575-85.

23. Overview of chemistry studies. In: Frances F, Marshall BD. A manual of laboratory and diagnostic tests. Lippincott Williams and Wilkins, and Wolters Kluwer Health (Pub) 2005 pp.338-482

*Corresponding author:

Dr Nidhi Rajendra. MD (Path), Assistant Professor, Department of Pathology, Dr.B R Ambedkar Medical College and Research Centre, KG Halli, Bangalore-65, Karnataka, India

Phone: +919535799579

Email: rnidhi25@gmail.com

Date of Submission : 04.12.2016

Financial or other Competing Interests: None.

Date of Acceptance : 02.01.2017

Date of Publication : 07.04.2017 